\title{
Redox State in Professional Cyclists Following Competitive Sports Activity
}

\author{
Roberto Corsetti ${ }^{1}$, Mauro Villa ${ }^{2}$, Massimo Pasturenzi ${ }^{2}$, Annarosa Finco ${ }^{*}, 3$ and Umberto Cornelli ${ }^{4}$ \\ ${ }^{I}$ Liquigas Cannondale - Med Board, Sesto al Reghena, Italy \\ ${ }^{2}$ M2Omeo-Varese, Italy \\ ${ }^{3}$ Cor. Con. International Srl-Department of Oxidation Research-Parma, Strada Langhirano 264/1A, Parma (PR), Italy \\ ${ }^{4}$ Loyola University School of Medicine-Chicago, USA
}

\begin{abstract}
Introduction: Measuring oxidative stress (OS) and antioxidant reserve (AOR) may be useful in assessing the physical condition of athletes taking part in competitive sports activities.

Aim: During the "Giro d'Italia" in May 2011, the redox state of an entire cycle racing team was monitored to determine the risk of cyclists developing "redox inflammation", which might herald the onset of pathological conditions.

Materials and Methods: The nine members of the Liquigas cycle racing team were analyzed using a FRAS 4 Evolvo System. OS was determined by measuring plasma hydroperoxides (d-ROMs test) and AOR by measuring water-soluble antioxidants in the plasma (BAP test). The latter was expressed in terms of $\mu \mathrm{Eq}$ of Vitamin C. Both measurements were carried out at four different times during the race: baseline, in the morning two days before the start [B]; in the morning of the rest day after 9 stages [M1]; in the evening after the most stressing mountain stage [MS]; in the morning before the start of the last stage [M2]. Each cyclist's performance was determined at the end of the race according to an ordinal scale ranging from 1 to 9 .

Results: The d-ROMs test values $(\mathrm{M} \pm \mathrm{SD})$ increased over time from $245 \pm 17.2$ to $322 \pm 50.9$ CARR. U. (1 CARR.U. corresponds to $0.08 \mathrm{mg} / \mathrm{dL}$ of $\mathrm{H}_{2} \mathrm{O}_{2}$ ). The BAP test values dropped from 2574 to $1962 \mu \mathrm{Eq}$ of Vitamin C. Athletic performance was directly correlated with increases in OS (Spearman $r_{s}=0.714 p<0.05$ ), whereas no correlation was found with BAP $\left(r_{s}=0.143 \mathrm{p}>0.05\right)$.

Conclusions: Redox balance assessment is a useful tool to: a) monitor athletic performance and predict the onset of redox inflammatory state (RI), which might be detrimental to the athlete's physical condition; b) propose suitable supplementation with particular foods and dietary supplements.
\end{abstract}

Keywords: Cycling, d-ROMs test, BAP test, performance, redox inflammatory state.

\section{INTRODUCTION}

Research into the relationship between exercise and oxidative stress (OS) has shown that intense muscular contractile activity can result in the production of reactive oxygen species (ROS) [1, 2], and that protein oxidation limits muscle force and triggers fatigue. However ROS production should not be considered solely in negative terms, since reactive oxygen species are important in the adaptation mechanism and play a role in cell signaling [3]. This involves nuclear factor protein (NF-kB), mitogenactivated protein kinase (MAPK) $[4,5]$ and Nrf2/ARE (nuclear factor erythroid-like 2/antioxidant responsive element) and induces the gene transcription of many antioxidant protective enzymes, such as SOD (superoxide dismutase), catalase, glutathione peroxidase (GPx) and thioredoxin [6]. These antioxidant enzymes together with non enzymatic antioxidants make up the antioxidant reserve (AOR). When there is a balance between OS and the AOR,

*Address correspondence to this author at the Cor. Con. International SrlDepartment of Oxidation Research-Parma, Strada Langhirano 264/1A, Parma (PR), Italy; Tel: +390521 649157; Fax; +39 0521 397282;

E-mail: finco.annarosa@libero.it the condition can be defined as "passive OS" and immediate recovery after exercise is possible.

Strenuous physical exercise causes OS characterized by a sharp increase in lipid peroxidation products [7] that damages muscle mitochondrial membranes.

In this case, NF-kB and MAPK are excessively activated [8], which leads to Nrf2/ARE inhibition [9]. Consequently the reactive oxygen species are not counterbalanced by the antioxidant defense system. This imbalance triggers immune and inflammatory responses, and these initiate a catabolic condition [9] which may be defined as "active OS".

It is known that human skeletal muscle contraction contributes more than anything else to the exercise-induced increase in plasma interleukin-6 (IL-6) [10, 11], which is a marker of muscles reactivity and one of the most important myokines.

In an experiment performed on athletes immediately after an ultra-marathon foot race [12], an almost 800-fold increase in IL-6 serum levels was observed, and inflammatory parameters, such as serum amyloid (SAA) and C-reactive protein (CRP), were found to increase by two orders of magnitude. Forty-eight hours after the race, IL-6 levels had 
returned to normal, whereas SAA and CRP values were still extremely high. This indicates that the active OS was generating a condition that may be defined as a redox inflammatory state (RI).

One peculiar aspect of this condition was that the AOR had actually increased at the end of the race and was still high even forty-eight hours later, even though bilirubin and uric acid levels (which both have antioxidant activity) were the same as they were before the trial and red cell GSH concentrations were lower than before the trial [12]. This suggests that a RI state may be characterized by an increase in both oxidative markers and antioxidant capacity.

Therefore, to get a clear picture of the OS/AOR balance, it is necessary to measure both of them.

The aim of this trial was to monitor the OS/AOR ratio during the "Giro d'Italia" where athletes have to cover about $3,500 \mathrm{~km}$ (on the flat and up medium-steep and steep mountains) in 21 days. The Giro is one of the most strenuous bicycle races in the world.

\section{MATERIALS AND METHODS}

\section{Subjects}

All nine athletes in the Liquigas Team (see later) took part in the trial during the 2011 "Giro d'Italia". Each team member gave their informed consent to measurement of their oxidative balance.

The protocol was approved by the Italian Federation of Sports Medicine ethical committee.

\section{Characteristics of the 2011 "Giro D'Italia"}

The physical exertion required by athletes taking part in the "Giro d'Italia" is summarized in Table $\mathbf{1 .}$

The physical exertion required from the participants was extremely high. The winner took 84 hours to complete the Giro, at an average speed of about $42 \mathrm{~km} / \mathrm{h}$, and the worst performer completed the race at a speed of $>40.5 \mathrm{~km} / \mathrm{h}$.

\section{Food Intake}

Particular care was taken in the preparation of food for the team and the diet was based on ingredients typical of the Italian Mediterranean diet. The relevant data are shown in Table 2.

\section{Supplement with Antioxidant}

All the athletes were taking vitamin $\mathrm{C}$ far from the oxidative balance evaluation (in the evening before M1 and M2 stages and in the morning before the stage of MS) in order to have no interference with the evaluation. The vitamin $\mathrm{C}$ was taken in the amount of $500 \mathrm{mg}$ /day (the higher dosage allowed as food supplement by the Italian Regulatory Agency) as a slow release formulation.

\section{Redox Balance Analysis: OS/AOR}

Two common tests were used: the reactive oxygen metabolites (d-ROMs) test to measure OS and the biological antioxidant potential (BAP) test to measure the AOR. Both tests can be performed using the FRAS Evolvo System (H\&D srl-Parma Italy) and have been validated for clinical use $[13,14]$. Each test can be conducted in 5 minutes.
Table 1. Characteristics of the 2011 "Giro D'Italia" (2011, 729 May)

\begin{tabular}{|c|c|c|c|c|c|}
\hline \multirow{2}{*}{ Stage } & \multirow{2}{*}{ Type } & \multirow{2}{*}{$\mathbf{K m}$} & \multirow{2}{*}{$\begin{array}{l}\text { Range } \\
\mathbf{K m} / \mathbf{h}\end{array}$} & \multicolumn{2}{|c|}{ Climb \% } \\
\hline & & & & Mean & Mx \\
\hline 1 & Time trial & 19.3 & $51-55$ & - & - \\
\hline 2 & Flat $^{\mathrm{a}}$ & 244 & $38-42$ & 5.8 & 10.0 \\
\hline 3 & Flat $^{\mathrm{a}}$ & 173 & $38-42$ & 4.0 & 10.0 \\
\hline 4 & Medium mountain & 216 & $39-43$ & 3.8 & 18.0 \\
\hline 5 & Medium mountain & 191 & $37-41$ & 3.7 & 15.0 \\
\hline 6 & Flat & 216 & $38-42$ & 4.2 & 9.0 \\
\hline 7 & Medium mountain ${ }^{\mathrm{a}}$ & 110 & $36-40$ & 5.0 & 10.0 \\
\hline 8 & Flat & 217 & $37-41$ & - & - \\
\hline 9 & Mountain $^{\mathrm{a}}$ & 169 & $35-39$ & 6.1 & 12.0 \\
\hline \multicolumn{6}{|l|}{ Rest Day } \\
\hline 10 & Flat & 159 & $40-44$ & - & - \\
\hline 11 & Medium mountain & 144 & $37-41$ & 3.0 & 12.0 \\
\hline 12 & Flat & 184 & $40-44$ & - & - \\
\hline 13 & Mountain $^{\mathrm{a}}$ & 167 & $33-37$ & 4.1 & 14.0 \\
\hline 14 & Mountain $^{\mathrm{a}}$ & 210 & $33-37$ & 4.7 & 22.0 \\
\hline 15 & Mountain $^{\mathrm{a}}$ & 229 & $33-37$ & 6.5 & 18.0 \\
\hline \multicolumn{6}{|l|}{ Rest Day } \\
\hline 16 & Time trial $^{\mathrm{a}}$ & 12.7 & $24-28$ & 8.2 & 14.0 \\
\hline 17 & Medium mountain & 230 & $37-41$ & 3.1 & 10.0 \\
\hline 18 & Flat & 151 & $38-42$ & - & - \\
\hline 19 & Mountain $^{\mathrm{a}}$ & 209 & $34-38$ & 7.3 & 15.0 \\
\hline 20 & Mountain $^{\mathrm{a}}$ & 242 & $35-39$ & 3.9 & 14.0 \\
\hline 21 & Time trial & 31.5 & $48-52$ & - & - \\
\hline Total & & 3524.5 & & & \\
\hline Average & & & $37-41$ & 4.6 & 12.7 \\
\hline
\end{tabular}

${ }^{\mathrm{a}}$ Uphill run to the winning post.

The d-ROMs test measures plasma hydroperoxides concentrations [15]. Among the different reactive oxygen species (ROS) those having more importance in terms of modification of regulatory proteins are $\mathrm{H}_{2} \mathrm{O}_{2}$ (hydrogen peroxide), $\mathrm{ONOO}^{-}$(peroxynitrite) and $\mathrm{ROOH}$ (hydroperoxides) [16]. The measure of ROOH is one of the simplest and more sensible marker of oxidation [17] and they are largely of lipidic nature (therefore including isoprostanes), but they also consist of carbonylated proteins and oxidized nucleic acids. Because of this large valence $\mathrm{ROOH}$ can be consider a reliable method to measure the oxidation in plasma. d-ROMs values are expressed in CARR. U. (1 CARR.U. corresponds to $0.08 \mathrm{mg}$ of $\mathrm{H}_{2} \mathrm{O}_{2} / \mathrm{dL}$ ) and normal values range from 250 to 300 CARR.U.

Chemically, d-ROMs test is based on Fenton's reaction. The plasma hydroperoxides in an acid buffer oxidized a particular chromogen (N,N-diethyl-p-phenylenediamine) which becomes pink. The quantity of hydroperoxides in 
plasma sample is directly correlated with the color and revealed at $505 \mathrm{~nm}$ with the photometer FRAS Evolvo.

Table 2. Daily Food Intake During the Race

\begin{tabular}{|c|c|c|}
\hline & Type of Food & Quantity \\
\hline \multirow{10}{*}{ Breakfast } & Pasta $^{a}$ & $120 \mathrm{~g}$ \\
\hline & Orange juice (red) & $250 \mathrm{ml}$ \\
\hline & Soy milk & $250 \mathrm{ml}$ \\
\hline & Toasted wholemeal bread & $80 \mathrm{~g}$ \\
\hline & Cheese & $40 \mathrm{~g}$ \\
\hline & Omelette & $120 \mathrm{~g}$ \\
\hline & Ham & $60 \mathrm{~g}$ \\
\hline & Fruit jam & $40 \mathrm{~g}$ \\
\hline & Shelled nuts & $50 \mathrm{~g}$ \\
\hline & Coffee (decaff) & $50 \mathrm{ml}$ \\
\hline \multirow{6}{*}{ Snack During the Stage } & Bread & $150 \mathrm{~g}$ \\
\hline & Fruit jam & $40 \mathrm{~g}$ \\
\hline & Fruit & $250 \mathrm{~g}$ \\
\hline & Ham & $50 \mathrm{~g}$ \\
\hline & Sugar free (maltodextrin) jam & $120 \mathrm{~g}$ \\
\hline & Electrolyte supplement in water & $500 \mathrm{ml}$ \\
\hline \multirow{8}{*}{ Snack After the Stage } & Rice $^{a}$ & $150 \mathrm{~g}$ \\
\hline & Potatoes (boiled) & $500 \mathrm{~g}$ \\
\hline & Fruit cake & $200 \mathrm{~g}$ \\
\hline & Orange juice (red) & $500 \mathrm{ml}$ \\
\hline & Fruit & $400 \mathrm{~g}$ \\
\hline & Yogurt & $125 \mathrm{~g}$ \\
\hline & Shelled nuts & $20 \mathrm{~g}$ \\
\hline & Amino acid supplement & $10 \mathrm{~g}$ \\
\hline \multirow{4}{*}{ Dinner } & Rice or pasta ${ }^{a}$ & $200 \mathrm{~g}$ \\
\hline & Red meat & $300 \mathrm{~g}$ \\
\hline & Potatoes (boiled) & $450 \mathrm{~g}$ \\
\hline & Wholeweat bread & $50 \mathrm{~g}$ \\
\hline \multirow{2}{*}{ Snack Before Sleeping } & Yogurt & $125 \mathrm{~g}$ \\
\hline & Fruit & $250 \mathrm{~g}$ \\
\hline & Total daily energy intake & $25 \mathrm{Mj}$ \\
\hline
\end{tabular}

BAP test is based, instead, on the ability of a colored solution, containing ferric $\left(\mathrm{Fe}^{3+}\right)$ ions adequately bound to a special chromogenic substrate, to decolor when its $\mathrm{Fe}^{3+}$ ions are reduced to ferrous $\left(\mathrm{Fe}^{2+}\right)$ ions as well as it can be observed by adding a reducing system (plasma antioxidants). The discoloration is revealed at $505 \mathrm{~nm}$ with the photometer FRAS Evolvo after 5 minutes incubation.

BAP values are expressed as $\mu \mathrm{Eq}$ of Vitamin $\mathrm{C} / \mathrm{L}$, and mainly measure (but not exclusively) the water soluble AOR in plasma. Normal values range from 2200 to $2400 \mu \mathrm{Eq}$ of Vitamin $\mathrm{C} / \mathrm{L}$.

\section{Blood Sampling}

Blood samples were taken at different times as follow: the first, taken as "baseline [B]", was in the morning (9 am) of $5^{\text {th }}$ of May, two days before the start of the Giro; the second, defined as "monitoring 1 [M1]", was taken in the morning (9 am) of $16^{\text {th }}$ of May during a rest day; the third, defined as "after maximum stress [MS]", was taken in the evening (at $22 \mathrm{pm}$ ) on $28^{\text {th }}$ of May after the mountain stage (Sestriere); the fourth, defined as "monitoring 2 [M2]", was taken in the morning ( $9 \mathrm{am}$ ) before the start of the last stage.

Blood samples for the complete blood assessment were taken from the brachial vein in the quantity of $15 \mathrm{~mL}$ divided into three $5 \mathrm{~mL}$ tubes.

For d-ROMs and BAP test blood was collected though a fingertip, $0.15 \mathrm{~mL}$ were collected in a heparinized microcuvette. Plasma was isolated by centrifugation at $37^{\circ} \mathrm{C}$ for $60 \mathrm{sec}$, using the centrifuge which is part of the FRAS 4 Evolvo System. All the analyses were conducted within 30 minutes from blood collection and on fasting blood sample.

\section{Definition of Cut-Offs}

The proposed cut-off values defined for the performance of professional athletes should be considered arbitrary. Changes in d-ROMs and BAP test values have been widely seen in experiments in healthy "non-athlete" volunteers and in various types of disease (cardiovascular, cerebral, metabolic, etc.).

\section{d-ROMs Cut-Off}

d-ROMs values in normal subjects usually rise during and at the end of physical stress. We must therefore define a value which can be considered to be a "significant increase or reduction".

A $>10 \%$ change in d-ROMs test value was considered to be a significant change beyond the limits of individual variability. Based on experience with healthy "non-athlete" volunteers with average values of about 250 CARR. U., this variability does not usually exceed 5\% [15].

The level of hydroperoxides measured with the d-ROMs test has been found to be correlated with hs-CRP [18]. In case of values out of the cut-off, d-ROMs may indicate an inflammatory condition which can be defined as "redox inflammatory".

\section{BAP Cut-Off}

The BAP test result of a physically fit subject under stress should remain stable or increase slightly. This is not the case in subjects who are not physically fit, where the AOR is depleted more easily.

In a sporting context, a change of $>10 \%$ was considered to be a significant variation, well beyond the limits of individual variability. Based on experience with healthy nonathlete volunteers, individual variability does not usually exceed 5\% [internal data].

A condition characterized by an increase in the d-ROMs value together with a reduction in the AOR indicates the 
onset of an imbalance which may lead to RI type responses and herald pathological phenomena.

\section{Other Variables for Lab Assessment}

The following analysis were carried out: blood cells counts, electrolytes, Hct, hs-CRP, blood glucose, lipids profile (total cholesterol, HDL, LDL, VLDL, triglycerides), $\mathrm{CK}, \mathrm{Hb}$ and urine analysis.

\section{Overall Performance Assessment}

The performance value was based on the overall judgment of the team doctor and coach. At the end of each stage, the performance of each athlete was scored between 1 (bad performance) and 4 (very good performance) and, at the end of the tour, the total performance score was obtain by the sum of each stage score. These final values of each athlete were ordered according to an ordinal scale from 1 to 9 , where 9 represents the best and 1 the poorest performance. The technical team and the medical team did not know the oxidative status of the athletes and considered all the aspects of athlete performance in relation to the health condition.

Performance was assessed as an overall value and did not depend on the general classification, which - in the strategy of a major stage race - does not in any way reflect the performance of the individuals. Athletes who have to perform certain tasks for the team and captain during the first stages of the race often reach the winning post way behind everyone else, and are therefore among the last in the general classification. However they might be judged to have performed successfully because their performance did not disappoint the expectations of the technical and management team.

It is also evident that parts of the performance or, on some occasions, the entire performance may be negatively influenced by state of health (as was the case with athlete n. 8).

\section{Statistical Analysis}

The mean and standard deviation (SD) were calculated for the two variables studied. The repeated measure ANOVA followed by the Tukey-Kramer multiple-comparison test was applied to determine the differences among the determinations. The percentage increases or a decrease with respect to the baseline (B) was also calculated for the values of the variables at M1, MS and M2. Recovery after MS was measured considering the percentage difference between MS and $\mathrm{M} 2$.

Non-parametric Spearman correlation coefficients $\left(r_{s}\right)$ were calculated between changes in d-ROMs and BAP test values vs. baseline (M1-B, M2-B and MS-B) and overall performance.

\section{RESULTS}

\section{Summary of the Salient Data on the Athletes During the Giro}

Athlete 1: he suffered from recurrent perineal furunculosis, and lumbago limited to the periods of intense stress during the climb in certain stages halfway through the Giro (he suffered from two small hernias documented by MRI).
Athlete 2: he suffered first from tracheitis then catarrhal bronchitis during the last stages of the race, from the stage ending at San Pellegrino Terme onwards. He was treated with azithromycin, acetylsalicylic acid and $\mathrm{N}$-acetylcysteine, and improved just enough to finish the race. Clarithromycin was added to the recommended treatment the day before the end of the race, due to his condition worsening with bronchial secretions.

Athlete 3: he suffered from small shooting pains in the right subcostal region throughout the Giro. He experienced them occasionally during intense stress (normal transaminase levels at the beginning of the Giro). When most severe (but never limiting), he took butylscopolamine tablets to relieve the pain. He will be undergoing ultrasonography in the next few days. Similar symptoms three years ago were attributed to biliary sand in the gallbladder.

Athlete 4: he suffered from a couple of fleeting episodes of allergic rhinoconjunctivitis kept successfully under control by levocitrizine and montelukast administered in the evening before going to bed, but only on a few particular occasions and far from the oxidative balance evaluation. He developed herpes labialis halfway through the Giro, which was treated with a local antiviral agent and protective total sun screen.

Athlete 5: he fell during the Orvieto stage and suffered grazes in the left and right hip, right thigh and right elbow regions. As he was leaving the hotel on the day of the Belluno-Nevegal time climb, he had an accident and suffered a lacerated contused scalp injury, which was treated without requiring stitches. He suffered from minor bronchial secretions about halfway through the Giro.

Athlete 6: he had cough and catarrh right from the first stages of the race. Mucolytics did not greatly improve the situation, which worsened, and he began antibiotic treatment with clarithromycin twice a day for several days. During the last few days of antibiotic treatment, the athlete reported episodes of diarrhoea on and off during both the rest day and the subsequent stages.

Athlete 7: he was in good condition, fit and healthy. The Giro offered him very few opportunities. He did not manage to reach the heart of the pack during the very few final sprints, which he watched from behind the sprinters competing for victory. He almost always finished between fifth and 15 th place. He did not struggle during the climbs and never needed to tag onto the "autobus" to tackle the toughest slopes. In practice, he is a sprinter. However, he admitted he has lost something of his explosiveness.

Athlete 8: he suffered from catarrhal tracheobronchitis halfway through the Giro, which became increasingly severe. He was treated with acetylsalicylic acid and azithromycin (for three days). There was a decisive improvement in the situation, but a few secretions remained to keep him company until the end of the Giro.

Athlete 9: he suffered from an episode of nausea with vomiting the night before the start of the race, on the evening of the initial team time trials. He suffered from perineal furunculosis during the first few days, although not severe. He suffered from a catarrhal cough from the stage ending at San Pellegrino Terme (which he won) onwards, which was treated with acetylsalicylic acid and N-acetyl-cysteine. He 
Table 3. Variables Measured at the Four Observation Times: Data for the Individual Athletes (Mean \pm SD and ANOVA)

\begin{tabular}{|c|c|c|c|c|c|c|c|c|}
\hline Athlete No. & \multicolumn{8}{|c|}{ Times } \\
\hline 1 & 240 & 2598 & 240 & 2780 & 303 & 2580 & 288 & 1821 \\
\hline 2 & 256 & 2160 & 303 & 2615 & 381 & 2184 & 396 & 2664 \\
\hline 4 & 256 & 2465 & 312 & 2210 & & & 312 & 2090 \\
\hline 5 & 256 & 3430 & 350 & 2630 & 389 & 3106 & 334 & 1749 \\
\hline 6 & 218 & 2605 & 334 & 2028 & 303 & 2640 & 288 & 1835 \\
\hline 7 & 233 & 2243 & 256 & 2376 & 312 & 2755 & 264 & 1930 \\
\hline SD & 17.2 & 383.6 & 37.1 & 249.8 & 50.9 & 347.3 & 42.6 & 302.8 \\
\hline ANOVA $^{c}$ & & & $\mathrm{P}<0.01$ & n.s. ${ }^{*}$ & $\mathrm{P}<0.01$ & n.s.* & $\mathrm{P}<0.01$ & $\mathrm{P}<0.05$ \\
\hline Other Variables & \multicolumn{2}{|c|}{$\begin{array}{c}5 \text { May [B] } \\
\text { [Time } 9 \text { am] }\end{array}$} & \multicolumn{2}{|c|}{$\begin{array}{l}16 \text { May [M1] } \\
\text { [Time } 9 \text { am] }\end{array}$} & \multicolumn{2}{|c|}{$\begin{array}{l}28 \text { May [MS] } \\
\text { [Time } 22 \text { pm] }\end{array}$} & \multicolumn{2}{|c|}{$\begin{array}{l}29 \text { May [M2] } \\
\text { [Time } 9 \text { am] }\end{array}$} \\
\hline hs-CRP [mg/L] & \multicolumn{2}{|c|}{$1.4 \pm 3.21$} & \multicolumn{2}{|c|}{$1.3 \pm 0.62$} & \multicolumn{2}{|c|}{$2.4 \pm 2.46$} & \multicolumn{2}{|c|}{ n.d. } \\
\hline Leucocytes $\left[10^{3} / \mathrm{mL}\right]$ & \multicolumn{2}{|c|}{$5.2 \pm 1.20$} & \multicolumn{2}{|c|}{$5.7 \pm 1.59$} & \multicolumn{2}{|c|}{$4.6 \pm 0.48$} & \multicolumn{2}{|c|}{ n.d. } \\
\hline Hct $[\%]$ & \multicolumn{2}{|c|}{$42.7 \pm 1.55$} & \multicolumn{2}{|c|}{$39.5 \pm 1.59^{c}$} & \multicolumn{2}{|c|}{$40.5 \pm 2.34^{\mathrm{c}}$} & \multicolumn{2}{|c|}{ n.d. } \\
\hline $\mathrm{Hb}[\mathrm{g} / \mathrm{dL}]$ & \multicolumn{2}{|c|}{$14.5 \pm 0.66$} & \multicolumn{2}{|c|}{$13.2 \pm 0.63^{\mathrm{c}}$} & \multicolumn{2}{|c|}{$13.6 \pm 0.90^{\mathrm{c}}$} & \multicolumn{2}{|c|}{ n.d. } \\
\hline
\end{tabular}

${ }^{\mathrm{a}}$ Values calculated excluding athlete n. $8 ;{ }^{\mathrm{b}}$ Values calculated excluding athletes 4 and $8 ;{ }^{\mathrm{c}}$ ANOVA data compared with 5 May values [B] $\mathrm{p}<0.05 ;$ n.d. or not determined; ${ }^{*}$ n.s. or not significant.

reported that he felt unable to perform rapid changes of pace. The day after the end of the Giro, he was obliged to take azithromycin and clarithromycin due to the onset of fever.

The four measurements were performed on all the athletes with the exception of athlete n. 4, who skipped the measurement at MS (see Table 3).

Athlete n. 8 started the race with some symptoms of bronchitis and his oxidative balance was abnormal: his dROMs value was 365 CARR.U. and BAP value was 3960 $\mu \mathrm{Eq}$ of Vitamin $\mathrm{C}$ (compared with a mean value of 245 CARR.U. for the other athletes).

For this reason, he has been excluded from the final assessment, and the average values have been calculated only for the remaining eight athletes. The use of antibiotics and/or drugs became a necessity for some athletes but the administration of them was always far from the day of the 4 analysis (B, M1, M2, MS) in order avoid artifacts and never influence the oxidative balance.

\section{d-ROMs Test}

An increase in OS was observed in all athletes (with the exception of athlete n. 8).

As it is clear from Table $\mathbf{3}$, there was a progressive increase from 245 CARR.U. to 299 CARR.U. at M1 ( $p<$ $0.01 v s$ baseline), ultimately reaching 306 CARR.U. at M2 ( $\mathrm{p}<0.01 v s$ baseline1). After stage 20 - measured at MS only seven athletes were measured, and the average value
(322 CARR.U.) was the highest in the race $(\mathrm{p}<0.01$ vs baseline). However, the values determined at M1, M2 and Ms are not statistically different.

The last average team value measured showed a $24.9 \%$ increase in OS state, suggesting that some of the athletes may have been at risk of developing a redox inflammatory condition (also see the section regarding cut-off values).

The d-ROMs test values measured after MS were higher than those measured at the other times (322 CARR.U.). It should however be noted that the mean value for this time is not significantly higher ( $p>0.05)$ than the M1 value (299 CARR.U.). This is believed to be due to one value being missing (athlete n. 4) as well as the low levels of OS stemming from the good condition of most of the athletes.

\section{BAP Test}

The AOR can be seen to have decreased progressively from $2574 \mu \mathrm{Eq}$ to $2416 \mu \mathrm{Eq}$ at M1, ultimately reaching 1962 $\mu$ Eq of Vitamin C at M2 ( $<0.05 v s$ Baseline). The M2 value is equivalent to a mean decrease of $21.6 \%$ with respect to baseline values. An increase in the AOR was found in some cases, which cannot be interpreted as a healthy condition (see discussion). The differences between the BAP values at baseline and MS are not statistically significant ( $p>0.05)$.

The average BAP value was high $(2604 \boldsymbol{\mu E q})$ during the MS stage, but this data lends itself to contradictory interpretation since an increase beyond the cut-off $(10 \%)$ might indicate cellular damage. 


\section{Change in Cut-Off}

The percentage change in both d-ROMs and BAP values compared to baseline was used to determine the cut-off values. Table 4 gives the percentage changes observed in the $\mathrm{d}$-ROMs and BAP test results during the various stages of the race, as well as the recovery after stage 20 (End Value or EV defined as the recover after MS and M2), which was the most stressing stage (Sestriere).

The correlation with d-ROMs values calculated using the Spearman coefficient $r_{s}$ was $0.749(p<0.05)$, whereas no correlation was found with BAP test values $\left(r_{s} 0.143 p>\right.$ 0.05).

Most of the data are definitely beyond the cut-off both for d-ROMs increase and BAP decrease.

\section{Other Variables for Lab Assessment}

For hs-CRP, luecocytes, $\mathrm{Hct}$ and $\mathrm{Hb}$ the last measurement was at $22 \mathrm{pm}$ after the MS. The evaluation of the M2 was not done for these variables because any significant modification was expected $12 \mathrm{hrs}$ after the last measurement since these variables need much more time to recover. The blood cells count was normal in all the determinations, whereas hs-CRP was showing an average increase in the last two measures (Table 3 ) and the relative coefficient of variation (CV) was extremely high (between $48 \%$ to $230 \%$ ) did not allow any statistically reliable conclusions. However, the data were correlated with dROMs test ("r" between 0.759 and $0.764 ; p<0.05$ ) that was shown to anticipate the increase of hs-CRP, since some athletes with a normal hs-CRP showed values of d-ROMs test higher than 300 CARR.U. For what concerns the leucocyte counts a reduction was shown after the MS stage but the differences were not statistically significant compared to the baseline value. All the other variables (Hct and $\mathrm{Hb}$ ) were reduced significantly (ANOVA $\mathrm{p}<0,05$ ) with $\mathrm{CV}<7 \%$ that indicates a sufficient discriminating power.

Any significant correlation was found between d-ROMs and $\mathrm{Hct}$ or $\mathrm{Hb}$.

\section{DISCUSSION}

This study is limited by the small number of cases involved in the trial, but this is counterbalanced by repeatedly analyzing the same athletes during different types of performance.

This is the first time oxidative balance has been measured during a long term endurance cycle race.

Previous studies were conducted in similar conditions during the "Tour de France" [19]. Creatine kinase (CK) and aspartate aminotransferase (AAT) were taken as main variables and showed that the use of allopurinol is able to reduce the increase in both. Allopurinol is a xanthine oxidase (XO) inhibitor that reduces the overproduction of ROS caused by the metabolic transformation of hypoxanthine into xanthine during uric acid production.

In another bicycle race, where the athletes had to ride as many kilometres as possible in 24 hours [20], the same tests we chose (d-ROMs and BAP) were used to determine if diaphragmatic breathing could reduce the OS caused by endurance effort. Both d-ROMs and BAP test results increased in the control group, by about $50 \%$ and $20 \%$ respectively.

Our data shows that - on average - d-ROMs levels (ROS) increased and BAP levels (AOR) decreased as the race progressed, but both test results increased concomitantly in some athletes, which reveals the presence of a redox inflammatory condition that needs some explanation.

d-ROMs values increase during physical exercise due to a combination of reactive oxygen species that stem from three sources: mitochondria, metabolic transformation mainly driven by XO and NADPH oxidase, and neutrophil/ macrophage activation. In other words, ROS production can be defined as energy-related (mitochondria), metabolicrelated (XO and NADPH oxidase) and reactive-related (neutrophils and macrophages).

The energy pathway increase may be limited by the uncoupling effect [21], which is a common process that reduces ROS production. On the other hand, neither the

Table 4. Percentage Increases Compared to B (Baseline) or MS (Maximum Stress)

\begin{tabular}{|c|c|c|c|c|c|c|c|c|}
\hline \multirow{2}{*}{$\begin{array}{c}\text { Athlete } \\
\text { No. }\end{array}$} & \multicolumn{2}{|c|}{ M1 } & \multicolumn{2}{|c|}{ MS } & \multicolumn{2}{|c|}{ M2 } & \multicolumn{2}{|c|}{$\mathbf{E} \mathbf{V}^{\mathrm{a}}$} \\
\hline & d-ROMs & BAP & d-ROMs & BAP & d-ROMs & BAP & d-ROMs & BAP \\
\hline 1 & 0 & 7 & 26 & -1 & 20 & -30 & -5 & -29 \\
\hline 2 & 18 & $21^{*}$ & 49 & 1 & 55 & $23^{*}$ & 4 & $22 *$ \\
\hline 4 & 22 & -10 & & & 22 & -15 & & \\
\hline 5 & 37 & -23 & 52 & -9 & 30 & -49 & -14 & -44 \\
\hline 6 & 53 & -22 & 39 & 1 & 32 & -30 & -5 & -30 \\
\hline 7 & 10 & 6 & 34 & $23^{*}$ & 13 & -14 & -15 & -30 \\
\hline
\end{tabular}


metabolic nor the reactive production has a limitation related to ATP production for energy supply to muscles. As a consequence of this, the increase in hydroperoxides derives from three different pathways, two of which are not linked with muscular energy production. This means that when dROMs test results are high, to have a more precise indication of the athlete's condition, variables that can possibly reflect the involvement of the other two ROS producing pathways have to be analyzed. Biological antioxidant potential (BAP) may be used for this purpose.

Two possible causes of an excessive increase in BAP are: red cell haemolysis, which releases GSH into the plasma; and antioxidants deriving from endothelial cells (apoptosis) that are severely damaged by the aggression of neutrophils and macrophages. This might be the case of athlete n. 8 at the beginning of the race, or athlete n. 2 at the end of the race, who became seriously ill immediately after finishing the tour and needed antibiotic treatment.

In other words, an increase in BAP during endurance may be caused both by the stimulation of endogenous antioxidant reserve production and by an increase in cellular damage.

Following an increase in hydroperoxides, we should expect a decrease in the AOR to prevent OS from progressing. Once the AOR becomes excessively low, there are no more defenses against ROS, and cellular damage prevails leading to a RI. Therefore, the decrease in AOR is lower if the athletes are in good physical condition than it is if they are in poor condition.

This could be one of the reasons why there was no correlation between BAP and performance, whereas the correlation with d-ROMs was significant.

Serum AOR was shown to increase in athletes during an ultramarathon foot race (236 km with a 36 hour time limit). Their TAC (total antioxidant capacity) increased immediately after the race and was still about $30 \%$ higher than baseline even 48 hours after the end of the race. In the same experiment, uric acid and bilirubin levels returned to about normal after a significant increase at the end of the race. This indicates that the increase in AOR most probably stemmed from a tissue reaction produced by neutrophils and macrophages. This is confirmed by the concomitant rise in serum amyloid and C-reactive protein. This pattern is an example of a RI defined using classical inflammation parameters, which can be simply and quickly measured by following the athletes with a mobile laboratory during the various stages of the race.

The d-ROMs and BAP tests can be performed simultaneously in ten minutes with less than $0.1 \mathrm{ml}$ of blood from a finger tip and a portable device (FRAS Evolvo- H\&D srl- Parma-Italy): high quality reagents are added to $1 \mathrm{ml}$ disposable cuvettes. The measurements can then be repeated very simply. Both test seems to be relatively independent from $\mathrm{Hct}$ and $\mathrm{Hb}$. In the present trial no correlation was found among these variables and both d-ROMs and BAP. However, the limited number of athletes that have been analyzed does not allow detecting significant correlations between plasma volume modification and oxidative balance.
Once we have measured the athlete's oxidative balance, we need the tools to put it right if necessary.

Most of the time, eating food rich in antioxidants is not enough to reduce the effects of ROS production. There is no recipe that can be used for everyone because each athlete may need a tailor-made treatment with antioxidant supplements. However, we must avoid the mistake of using high doses of one or two single antioxidants because this can lead to OS, since all antioxidants at high doses become prooxidants. This was the case of athletes treated with large doses of both Vitamin E and Vitamin C during high-intensity endurance exercise training where it was clearly shown that MDA and carbonylated proteins were increased. This fact witnesses that a pro-oxidant effect was achieved instead of reducing the oxidative burden [11]

This suggests using a combination of physiological modulators [22] in low doses, since there are specific modulators that protect cell membranes, increase cytosol antioxidant capacity, support the production of antioxidant enzymes, and improve circulating antioxidant capacity [15] and the efficiency of erythrocytes [23].

In our trial, some of the athletes performed well even though their laboratory parameters indicated they were close to or in a redox inflammatory condition. This goes to show that muscles and physical fitness are only part of the performance and that the final result is determined by the athlete's will-power.

\section{CONCLUSION}

Cycling team athletes who have to perform a long term endurance event, such as the "Giro d'Italia", have to be followed carefully to monitor their health and performance in real time. This can only be done using a mobile laboratory to perform the various pertinent tests with quick results. For these reasons, simple and reliable tests that assess oxidative balance, such as the d-ROMs and BAP tests can be useful to give indications. Excessive OS could lead to a redox inflammatory condition that could worsen rapidly. The dROMs increase is evident even before the values of hs-CRP come out of the normality range and much before the clinical symptoms appear. In this experience modification of dROMs test also anticipates the hs-CRP increase in those athletes where it was found abnormal at the end of the tour. White blood cells count has been also determined and statistically no significant differences were found. Athletes may develop serious illnesses that might lead to dropping them from the race. The next step could be to analyze the support provided by physiological modulators - taken as supplements or through specific diets - in minimizing AOR loss and the impact of ROS on the performance and health of athletes.

\section{ACKNOWLEDGEMENTS}

This study was possible because of the contribution of the nine athletes and the staff composing the Liquigas Cannondale Team.

No financial or contractual agreement that may cause conflict of interest or be perceived as causing is in place. No works are being concurrently published or reviewed that are relevant to the manuscript. The corresponding author and all 
of the authors have read and approved the final submitted manuscript. No portion of this work has been or is currently under consideration for publication elsewhere.

No portion of this manuscript, other than the abstract, has been published or posted on the internet.

\section{CONFLICT OF INTEREST}

The authors confirm that this article content has no conflicts of interest.

\section{REFERENCES}

[1] Gomez-Cabrera MC, Borrás C, Pallardó FV, et al. Decreasing xanthine oxidase-mediated oxidative stress prevents useful cellular adaptations to exercise in rats. J Physiol 2005; 15( 567) (Pt 1): 11320.

[2] Radák Z, Pucsok J, Mecseki S, et al. Muscle soreness-induced reduction in force generation is accompanied by increased nitric oxide content and DNA damage in human skeletal muscle. Free Radic Biol Med 1999; 26(7-8): 1059-63.

[3] Reid MB. Invited Review: redox modulation of skeletal muscle contraction: what we know and what we don't. J Appl Physiol 2001; 90(2): 724-31.

[4] Gomez-Cabrera MC, Martínez A, Santangelo G, et al. Oxidative stress in marathon runners: interest of antioxidant supplementation. Br J Nutr 2006; 96(Suppl 1): S31-3.

[5] Ji LL. Modulation of skeletal muscle antioxidant defense by exercise: role of redox signaling. Free Radic Biol Med 2008; 44(2): $142-52$.

[6] Kobayashi M, Yamamoto M. Molecular mechanism activating the Nrf2-Keap1 pathway of antioxidant gene regulation. Antiox Redox Signal 2005; 7: 389-94.

[7] Viña J, Gomez-Cabrera MC, Lloret A, et al. Free radicals in exhaustive physical exercise: mechanism of production, and protection by antioxidants. IUBMB Life 2000; 50(4-5): 271-7.

[8] Ji LL, Gomez-Cabrera MC, Vina J. Exercise and hormesis: activation of cellular antioxidant signaling pathway. Ann N Y Acad Sci 2006; 1067: 425-35.

[9] Liu GH, Qu J, Shen X. NF-kB/p65 antagonizes Nrf2-ARE pathway by depriving $\mathrm{CPB}$ from $\mathrm{Nrf} 2$ and facilitating recruitment of HDAC3 to MafK. Biochem Biophys Acta 2008; 1783: 713-27.
[10] Ostrowski K, Rohde T, Zacho M, et al. Evidence that interleukin-6 is produced in human skeletal muscle during prolonged running. J Physiol 1998; 508( Pt 3): 949-53.

[11] Yfanti C, Fischer CP, Nielsen S, et al. Role of vitamin $\mathrm{C}$ and $\mathrm{E}$ supplementation on IL-6 in response to training. J Appl Physiol 2012; 112: 990-1000.

[12] Margeli A, Skenderi K, Tsironi M, et al. Dramatic elevations of interleukin- 6 and acute-phase reactants in athletes participating in the ultradistance foot race spartathlon: severe systemic inflammation and lipid and lipoprotein changes in protracted exercise. J Clin Endocrinol Metab 2005; 90(7): 3914-8.

[13] Nakayama K, Terawaki H, Nakayama M, et al. Reduction of serum antioxidative capacity during hemodialysis. Clin Exp Nephrol 2007; 11(3): 218-24

[14] Vassalle C. An easy and reliable automated method to estimate oxidative stress in the clinical setting. Methods Mol Biol 2008; 477: 31-9.

[15] Cornelli U, Terranova R, Luca S, et al. Bioavailability and antioxidant activity of some food supplements in men and women using the d-ROMs test as a marker of oxidative stress. J Nutr 2001; 132: 3208-11.

[16] Brigelius-Flohè R, Flohè L. Basic principles and emerging concepts in the redox control of transcription factors. Antiox Redox Signal 2011; 8: 2335-81

[17] Cornelli U, Belcaro G, Finco A. The oxidative stress balance measured in humans with different markers, following a single oral antioxidants supplementation or a diet poor of antioxidants. J Cosmet Dermatol Sci Appl 2011; 1: 64-70.

[18] Kamezaki F, Yamashita K, Kubara T, et al. Derivatives of reactive oxygen metabolites correlates with high-sensitivity C-reactive protein. J Atheroscler Thromb 2008; 15(4): 206-12.

[19] Gomez-Cabrera MC, Domenech E, Viña J. Moderate exercise is an antioxidant: upregulation of antioxidant genes by training. Free Radic Biol Med 2008; 44(2): 126-31.

[20] Martarelli D, Cocchioni M, Scuri S, et al. Diaphragmatic breathing reduces exercise-induced oxidative stress. Evid Based Complement Alternat Med 2011; 2011: 932430.

[21] Schrauwen P, Hesselink M. UCP2 and UCP3 in muscle controlling body metabolism. J Exp Biol 2002; 205(Pt 15): 2275-85.

[22] Olson JA. Benefits and liabilities of vitamin A and carotenoids. J Nutr 1996; 126(4 Suppl): 1208S-12S.

[23] Cornelli U. Treatment of Alzheimer's disease with a cholinesterase inhibitor combined with antioxidants. Neurodegener Dis 2010; 7(13): 193-202. 